\title{
MARKED DIFFERENCES IN LOCAL BONE REMODELLING IN RESPONSE TO DIFFERENT MARROW STIMULATION TECHNIQUES IN A LARGE ANIMAL
}

\author{
H.M. Zlotnick ${ }^{1,2,3}$, R.C. Locke ${ }^{1,3}$, B.D. Stoeckl ${ }^{1,2,3}$, J.M. Patel ${ }^{1,3,4}$, S. Gupta ${ }^{1,3}$, K.D. Browne ${ }^{5}$, J. Koh ${ }^{6}$, \\ J.L. Carey ${ }^{1,3}$ and R.L. Mauck ${ }^{1,2,3, *}$ \\ ${ }^{1}$ McKay Orthopaedic Research Laboratory, Department of Orthopaedic Surgery, \\ University of Pennsylvania, Philadelphia, PA, USA \\ ${ }^{2}$ Department of Bioengineering, University of Pennsylvania, Philadelphia, PA, USA \\ ${ }^{3}$ Translational Musculoskeletal Research Center, CMC VA Medical Center, Philadelphia, PA, USA \\ ${ }^{4}$ Department of Orthopaedics, Emory University, Atlanta, GA, USA \\ ${ }^{5}$ Center for Neurotrauma, Neurodegeneration and Restoration, CMC VA Medical Centre, \\ Philadelphia, PA, USA \\ ${ }^{6}$ Orthopaedic and Spine Institute, NorthShore University Health System, Evanston, IL, USA
}

\begin{abstract}
Marrow stimulation, including subchondral drilling and microfracture, is the most commonly performed cartilage repair strategy, whereby the subchondral bone plate is perforated to release marrow-derived cells into a cartilage defect to initiate repair. Novel scaffolds and therapeutics are being designed to enhance and extend the positive short-term outcomes of this marrow stimulation. However, the translation of these newer treatments is hindered by bony abnormalities, including bone resorption, intralesional osteophytes, and bone cysts, that can arise after marrow stimulation. In this study, three different marrow stimulation approaches - microfracture, subchondral drilling and needle-puncture - were evaluated in a translationally relevant large-animal model, the Yucatan minipig. The objective of the study was to determine which method of marrow access (malleted awl, drilled Kirschner wire or spring-loaded needle) best preserved the underlying subchondral bone. Fluorochrome labels were injected at the time of surgery and 2 weeks postsurgery to capture bone remodelling over the first 4 weeks. Comprehensive outcome measures included cartilage indentation testing, histological grading, microcomputed tomography and fluorochrome imaging. Findings indicated that needle-puncture devices best preserved the underlying subchondral bone relative to other marrow access approaches. This may relate to the degree of bony compaction occurring with marrow access, as the Kirschner wire approach, which consolidated bone the most, induced the most significant bone damage with marrow stimulation. This study provided basic scientific evidence in support of updated marrow stimulation techniques for preclinical and clinical practice.
\end{abstract}

Keywords: Bone remodelling, fluorochrome, large-animal models, microfracture, marrow stimulation, drilling.

*Address for correspondence: Robert L. Mauck, 308A Stemmler Hall, 3450 Hamilton Walk, Philadelphia, PA, 19104-6081, USA.

Telephone number: +1 2158988653 Fax number: +1 2155732133 Email: lemauck@pennmedicine.upenn.edu

Copyright policy: This article is distributed in accordance with Creative Commons Attribution Licence (http://creativecommons.org/licenses/by-sa/4.0/).

\begin{tabular}{|c|c|c|c|}
\hline & List of Abbreviations & K-wire & Kirschner wire \\
\hline \multirow{2}{*}{ AMIC } & autologous matrix-induced & $\mathrm{OA}$ & osteoarthritis \\
\hline & chondrogenesis & OCT & optimal cutting temperature \\
\hline ANOVA & analysis of variance & PBS & phosphate-buffered saline \\
\hline BV/TV & bone volume/total volume & PMMA & polymethyl methacrylate \\
\hline IACUC & Institutional Animal Care and Use & $\mathrm{SafO} / \mathrm{FG}$ & safranin $\mathrm{O} /$ fast green \\
\hline & Committee & TRAP & tartrate-resistant phosphatase \\
\hline ICRS & $\begin{array}{l}\text { International Cartilage Repair } \\
\text { Society }\end{array}$ & $\mu \mathrm{CT}$ & micro-computed tomography \\
\hline
\end{tabular}




\section{Introduction}

Focal cartilage injuries are common, impacting $\sim 1$ million Americans annually (Mithoefer et al., 2009). Due to the poor intrinsic healing capability of articular cartilage, such lesions may progress to degenerative OA if left untreated (Heijink et al., 2012). In the United States alone, OA impacts over 27 million people, resulting in over 100 billion US dollars of healthcare costs (Jafarzadeh and Felson, 2018; Murphy and Helmick, 2012). With severe OA, joint arthroplasty may be the only option to restore mobility and improve life quality.

In an effort to repair cartilage defects, and ultimately prevent joint-wide $\mathrm{OA}$, the most common procedure is MST (Martín et al., 2019). MST is a relatively simple and cost-effective strategy to introduce regenerative cells into a cartilage defect. The process involves the initial debridement of the cartilage defect, followed by perforation of the subchondral bone plate using either a fluted drill bit, K-wire (subchondral drilling) or surgical awl (microfracture) (Gao et al., 2018; Steadman et al., 2002). Then, the pressure within the marrow compartment promotes marrow-derived fluid and cells to populate the cartilage defect, form a clot and serve as a template for the formation of a fibrous matrix (DiBartola et al., 2016).

While MST primarily leads to fibrous tissue formation (Knutsen et al., 2004), it remains an important first-line treatment for cartilage defect repair. MST can be performed as a single arthroscopic procedure and has been shown to improve knee function in 70-95\% of patients (assessed 2-11 years post-surgery) (Gobbi et al., 2005; Knutsen et al., 2007; Steadman et al., 2003). To further improve and extend these outcomes, MST has been combined with a collagen type I/III membrane to engage and hold the marrow clot in place (Volz et al., 2017). This procedure is referred to as AMIC and has shown favourable results in comparison to MST alone 5 years postsurgery. In addition to AMIC, several laboratories have developed technologies focused on augmenting MST to direct cell differentiation and produce hyaline-like cartilage (Kim et al., 2015; Madry et al., 2020; Miller et al., 2014; Morisset et al., 2007; Patel et al., 2020; Sennett et al., 2021; Zanotto et al., 2019). Only a few of these strategies have progressed beyond the preclinical testing stage (Sharma et al., 2013).

Given the role of the subchondral bone in OA, it is likely that for augmented MST to reach its full potential, steps must be taken to address unintentional bony abnormalities that may arise from marrow access. This includes reports of bone resorption, intralesional osteophytes and the formation of bone cysts (Cole et al., 2011; Zedde et al., 2016). These subchondral irregularities likely impede the translation of novel scaffolds and therapeutics (Sennett et al., 2021) and may also limit revision treatment options in the case of MST failure (Riff et al., 2020). Intuitively, without a stable foundation on which to form, the repair tissue may be predisposed to failure. To reduce bone damage, it may be beneficial to scale down tool sizing. Indeed, both small (1 mm) diameter awls (Orth et al., 2016) and small $(1 \mathrm{~mm})$ diameter $\mathrm{K}$-wires have recently been shown to improve MST outcomes in comparison to larger instrumentation (Eldracher et al., 2014). Others have suggested that small diameter $(1 \mathrm{~mm})$ holes that are deeper ( $9 \mathrm{~mm}$ depth) may preserve the underlying bone in comparison to more traditionally larger ( $2 \mathrm{~mm}$ ) and shallower (2-4 $\mathrm{mm})$ holes that can fragment and compact the bone (Chen et al., 2009; Zedde et al., 2016).

While there is growing evidence to support the use of smaller diameter and deeper access for MST, the mechanism for creating these narrow, extended holes (whether it be by impaction, drilling or another method) is not yet determined. To date, only 7 studies have directly compared the standard MST approaches - microfracture and subchondral drilling - and only 3 of these studies included multiple outcome measures (Kraeutler et al., 2020). Thus, there is a lack of knowledge regarding how the method of hole creation impacts the subchondral bone. Therefore, the goal of this study was to evaluate the impact of 3 different methods of MST (awl-based microfracture, needle puncture and drilling) on the underlying subchondral bone in a Yucatan minipig model of cartilage defect repair. The needle puncture device (SmartShot ${ }^{\circledR}$ marrow access device, Marrow Access Technologies, Minnetonka, MN, USA) employed is spring-powered and self-retracting and, therefore, different from the awl in the consistency and speed of entry and exit from the bone and its overall penetration depth into the bone $(\sim 4 \times$ deeper than an awl). For this study, one SmartShot ${ }^{\circledR}$ device size-matched in diameter to the microfracture awl and another SmartShot ${ }^{\circledR}$ device size-matched in diameter to the K-wire were included, resulting in 4 experimental treatment groups for comparison. The study hypothesis was that the smaller SmartShot ${ }^{\circledR}$ device $(0.9 \mathrm{~mm}$ diameter $)$ would cause the least bone resorption and compaction, as assessed 4 weeks post-MST. Also, bone fluorochrome labelling was implemented to track bone remodelling over the study duration. This technique was first pioneered by Dr Berton Rahn and Dr Stephen Perren (Rahn and Perren, 1971) and provided a unique window into the dynamics of bone healing at these MST marrow access sites.

\section{Materials and Methods}

\section{Animal study}

All animal procedures were approved by the IACUC at the University of Pennsylvania. 6 skeletally mature (age 12 months at the beginning of the study) male Yucatan minipigs (Sinclair Bioresources, Auxvasse, MO, USA) were used. Under a single anaesthetic induction, two separate surgical procedures were 
performed (Fig. 1). Both the left upper extremity and right stifle joint were sterilely draped and prepared using betadine. In the first procedure, an indwelling catheter was implanted in the brachiocephalic vein. In a second procedure, a unilateral stifle joint surgery was performed using a minimally invasive open approach (Bonadio et al., 2017). During the stifle joint surgery, a bone fluorochrome label was infused through the implanted catheter. 2 weeks post-surgery, a second bone fluorochrome label was infused to track bone remodelling. Animals were euthanised 4 weeks postsurgery. Cartilage indentation testing, $\mu \mathrm{CT}$ (bone resorption), mineralised cryohistology (fluorochrome labelling, tartrate resistant phosphatase staining) and demineralised paraffin-wax histology (SafO/FG and haematoxylin and eosin stains) with blinded ICRS II scoring were performed on the defects from the operative limbs post-euthanasia. Four of the nonoperative limbs were used for a time 0 assessment of bone compaction by $\mu \mathrm{CT}$.

\section{Catheter implantation}

For the catheter implantation, a $4 \mathrm{~cm}$ incision was made in the left neck $4.5 \mathrm{~cm}$ lateral to the manubrium. Dissection was carried down to the brachiocephalic vein. Medial and lateral ties (0 silk) were loosely tied to the vein. A cannula and a trocar were driven subcutaneously from the neck incision to the left scapula. The trocar was removed and the catheter (79 cm length, Luer lock, SAI Infusion Technologies, Lake Villa, IL, USA, CPJC-12) was passed through the cannula, leaving the Luer hub exteriorised at the left scapula. An 11-blade was used to create an opening in the brachiocephalic vein for passage of the catheter. The silk ties were fastened to secure the catheter to the vein. Both the neck and scapular incisions were closed and the catheter was flushed to assure its function.

\section{Stifle joint surgery}

Immediately after closing the incisions from the catheter implantation, a unilateral stifle joint procedure was performed on the right hind limb. A medial parapatellar skin incision exposed the trochlea. Six full-thickness chondral defects were created in the trochlear groove using a $5 \mathrm{~mm}$ biopsy punch. A curette was used to excise cartilage tissue from within the bounds of the scored defect. Care was taken to preserve the subchondral plate. In each animal, a single defect was left untreated (empty) as a control $(n=6)$. Treatment groups included: awl-based microfracture (diameter: $0.8 \mathrm{~mm}$, depth: $2 \mathrm{~mm}$ ), SmartShot ${ }^{\circledR}$ (diameter: $0.9 \mathrm{~mm}$, depth: $8 \mathrm{~mm}$ ), SmartShot ${ }^{\circledR}$ (diameter: $1.2 \mathrm{~mm}$, depth: $8 \mathrm{~mm}$ ) and drilling with a K-wire (diameter: $1.25 \mathrm{~mm}$, depth: $6 \mathrm{~mm}$ ). For each treatment, 3 MST holes were created, except for the SmartShot ${ }^{\circledR}(1.2 \mathrm{~mm})$ where the outer housing limited the hole number to 2 . The 4 treatment groups were positionally randomised across 28 defects ( $n=7 /$ treatment group). All animals received at least 1 of each treatment group. 2 defects were used for another study (36 total defects across the 6 animals). Sample size was determined based on

\section{a Study timeline}

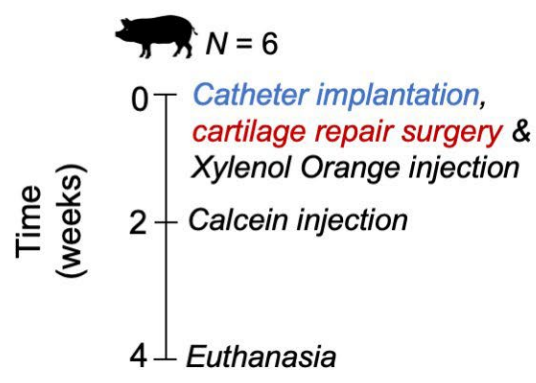

b Surgical instruments

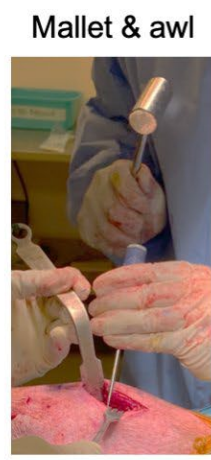

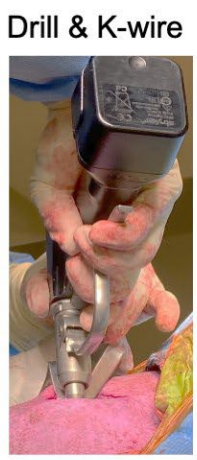

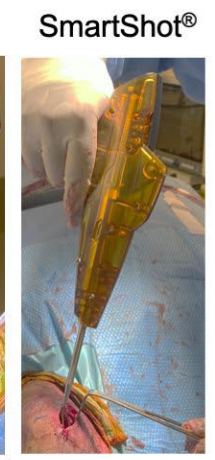

\section{c Surgical procedures}
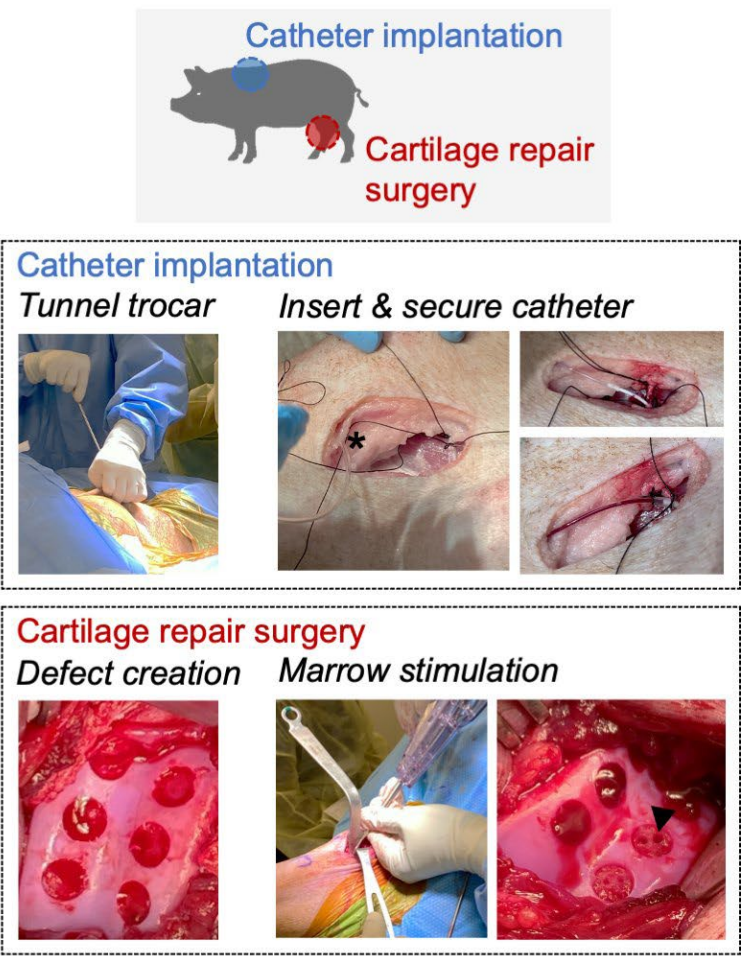

Fig 1. Study overview. (a) Study timeline. (b) Surgical instruments for cartilage repair procedure. (c) Surgical procedures. Catheter implantation: an indwelling catheter was implanted in the brachiocephalic vein for fluorochrome delivery. ${ }^{*}$ denotes tunnelled catheter. Cartilage repair surgery: six full-thickness chondral defects (5 mm diameter) were created in the trochlea of the stifle joint. Arrowhead: MST hole. 
an a priori power analysis using previous data in this animal model (Pfeifer et al., 2017).

\section{Fluorochrome injections and catheter maintenance}

Xylenol orange (90 mg/kg, Millipore, 398197) was injected slowly over $1 \mathrm{~h}$ through the indwelling catheter during the stifle joint procedure (Funk et al., 2009). Xylenol orange was dissolved in $100 \mathrm{~mL}$ saline (Medline, Wilmer, TX, USA, EMZ111240) and sterile filtered. 2 weeks post-surgery, the animals were sedated, anaesthetised and intubated. Under anaesthesia, calcein (15 mg/kg, Millipore, C0875) was injected slowly over $1 \mathrm{~h}$ through the indwelling catheter (Funk et al., 2009). Calcein was dissolved in $100 \mathrm{~mL}$ of $1.4 \%$ (wt/vol) sodium bicarbonate (Millipore, S5761) and sterile filtered. Blood draws were performed through the catheter before each fluorochrome injection, 1 min post-injection, $1 \mathrm{~d}$ post-injection and 1 week post-injection. Blood samples were analysed to measure ionised calcium (Antech Diagnostics, New Hyde Park, NY, USA). The catheters were flushed twice daily with saline and heparin locked (100 U/mL, Medsupply Partners, Atlanta, GA, USA, BD-306423) to maintain patency. One animal's catheter was not patent at the $1 \mathrm{~d}$ time point after the calcein injection, decreasing the sample size to 5 animals for the $1 \mathrm{~d}$ and 1 week time points. All animals wore Thundershirts for dogs (Thunderworks, Durham, NC, USA, BSSXXL-T01) to protect the exteriorised catheter hub and prevent pull-out.

Euthanasia and cadaveric use of non-operative stifle joint All animals were euthanised 4 weeks post-surgery. The operative limbs were prepared for cartilage indentation testing. 4 of the non-operative contralateral stifle joints (left hind limbs) were used to assess initial (time 0) local bone compaction surrounding the MST holes. For this assessment, mock surgeries were performed on the cadaveric joints, mimicking the operative limbs. Briefly, chondral defects ( $n=6$ defects $/ \mathrm{knee}$ ) were created using a $5 \mathrm{~mm}$ biopsy punch and curette. Each limb had one nontreated (empty) defect control ( $n=4$ empty defects) and the surgical treatments [microfracture $(0.8 \mathrm{~mm})$, SmartShot ${ }^{\circledR}(0.9 \mathrm{~mm})$, SmartShot $^{\circledR}(1.2 \mathrm{~mm}), \mathrm{K}$-wire $(1.25 \mathrm{~mm})]$ were randomised to the remaining defects ( $n=5$ defects/treatment).

\section{Cartilage indentation testing}

Following euthanasia, a hand saw was used to produce $1 \mathrm{~cm}^{3}$ osteochondral units containing the chondral defect from the operative limbs (Sennett et al., 2021). Control cartilage was collected from the distal end of the trochlea. Each sample ( $n=6-7$ defects/group) was potted in PMMA (OrthoJet, Lang Dental, Wheeling, IL, USA) to secure the bone. All samples were tested. Samples were covered in PBS with protease inhibitors (Roche Complete, Millipore) and tested within $5 \mathrm{~h}$ of collection. Each sample was placed in a custom rig equipped with an $X Y$ positioning stage and a goniometer to ensure that the cartilage surface was indented perpendicular to the $2 \mathrm{~mm}$ diameter spherical indenter (Meloni et al., 2017). A load of $0.1 \mathrm{~N}$ was applied at an initial rate of $0.1 \mathrm{~mm} / \mathrm{s}$ and held over $900 \mathrm{~s}$ as the tissue underwent creep displacement. The compressive modulus $\left(E_{y-}\right)$ was computed by fitting the creep data to a Hertzian Biphasic analytical model (Moore et al., 2016). Cartilage thickness was experimentally calculated from samples excised during surgery and input into the analytical model.

\section{$\mu \mathrm{CT}$}

After indentation testing, all samples ( $n=7$ defects/ group) were removed from the PMMA, submerged in PBS with protease inhibitors and imaged by

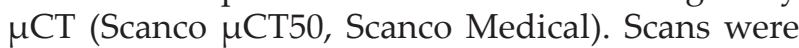
conducted utilising the following parameters: 1,500 projections, $851 \mathrm{~ms}$ per 1 exposures/projection, voltage $70 \mathrm{kVp}$, current $85 \mu \mathrm{A}$, isotropic voxel size $10.3 \mu \mathrm{m}$. The volume of bone resorption was calculated using the Scanco evaluation software by manually contouring the void space underneath the neocartilage. The MST holes were excluded from these measurements.

Parallel $\mu \mathrm{CT}$ scans were performed on the osteochondral units from the cadaveric time 0 surgeries using the contralateral hind limbs. The same $\mu \mathrm{CT}$ imaging parameters were used for these samples. For each treatment condition, the local BV/ TV surrounding individual MST holes $(n=6$ holes analysed/treatment) was calculated. Using the Scanco evaluation software, an outer circle was drawn with a diameter $0.65 \mathrm{~mm}$ longer than the original hole diameter. This value was selected to ensure that the volume measured was unique to that hole and not overlapping with another MST hole within the same defect. Subsequently, the perimeter of the MST hole was contoured within this outer circle, creating a hollow cylinder. The BV/TV was computed for this hollow cylinder, excluding the inner void space.

\section{Histology}

Mineralised cryohistology

Samples were fixed immediately after $\mu \mathrm{CT}$ scanning for $24 \mathrm{~h}$ in $10 \%$ neutral buffered formalin. After fixing, samples were infiltrated for $48 \mathrm{~h}$ with a $10 \%$ sucrose (Fisher Scientific, S25590) and $2 \%$ polyvinylpyrrolidone (Millipore, P5288) solution. Then, samples were embedded in OCT compound and sectioned (18 $\mu \mathrm{m} / \mathrm{section})$ undecalcified using cryofilm until reaching the midplane of the defect using a motorised cryostat (CM1950, Leica) (Dyment et al., 2016). Tape-stabilised, frozen sections were subjected to two rounds of imaging using a Zeiss Axio Scan.Z1 digital slide scanner, including imaging of (i) fluorochrome labels and dark field, (ii) TRAP staining with TO-PRO-3 Iodide (Scientific, T3605) counterstain. For TRAP staining, sections were incubated for $1 \mathrm{~h}$ in TRAP buffer $(0.92 \%$ sodium acetate anhydrous, $1.14 \% \mathrm{~L}_{(+)}$-tartaric acid, $1 \%$ glacial 
acetic acid $\mathrm{pH}$ 4.1-4.3) and then incubated for $1 \mathrm{~h}$ under ultraviolet light with ELF97 substrate (Thermo Fisher Scientific, E6588) in TRAP buffer.

Fluorochrome labels (\% area) were quantified in the region surrounding the MST holes. Images were split into red and green channels, representing the xylenol orange and calcein signals, respectively. Then, images were binarised and rotated in Fiji to orient the MST holes perpendicular to the surface (Schindelin et al., 2012). A region of interest (3 $\mathrm{mm}$ by $1 \mathrm{~mm}$ ) was defined immediately adjacent to the MST hole. Sample size ( $n=6-7$ holes/treatment) was determined by the number of distinct sections with an apparent MST hole. Only one hole was measured for a given defect. TRAP staining ( $n=5-6$ defects/ treatment) was quantified by measuring the number of positive TRAP pixels within a $7 \mathrm{~mm}$ by $7 \mathrm{~mm}$ area normalised by the bone area within this region.

\section{Demineralised paraffin-wax histology}

After cryosectioning one half of each defect, all samples ( $n=7$ defects/group) were removed from OCT and decalcified (Formical 2000, StatLab Medical Products) for $2 \mathrm{~d}$. Then, these decalcified samples were processed for paraffin-wax histology. Sections from the midplane of each defect were cut $(7 \mu \mathrm{m})$ using a paraffin microtome (RM2245, Leica) and stained with haematoxylin and eosin and SafO/FG.

\section{Histological scoring}

SafO/FG-stained sections were assessed using the modified ICRS II scoring system (Mainil-Varlet et al., 2010). The following parameters were scored on a continuous scale from 0 (poor defect repair) to 100 (normal articular cartilage): defect fill, integration to surrounding cartilage, matrix staining, surface architecture, basal integration, subchondral bone abnormality, vascularisation, surface/superficial assessment, mid/deep zone assessment, overall assessment. Three blinded reviewers with expertise in cartilage histomorphology scored each sample. Scores for each defect were averaged and plotted by treatment group.

\section{Data analysis}

All quantitative data were analysed using GraphPad Prism (version 8.4.3 for MacOS, GraphPad Software). For mechanical testing, histological scoring, blood analyses, fluorochrome labelling, bone resorption and TRAP data, a one-way ANOVA was performed with post-hoc Tukey's corrections for multiple comparisons. For the bone compaction analysis, a Kruskal-Wallis test was performed with a post-hoc Dunn's test. All plots were made in Prism.

\section{Results}

\section{Surgical outcomes and blood analyses}

All animal procedures were performed without complications (Fig. 1) and animals were allowed weight bearing within $2 \mathrm{~h}$ post-surgery. Animal skin coloration changed dramatically immediately after fluorochrome injection (Fig. 2a), evidence of

a Minipig coloring post-fluorochrome injection
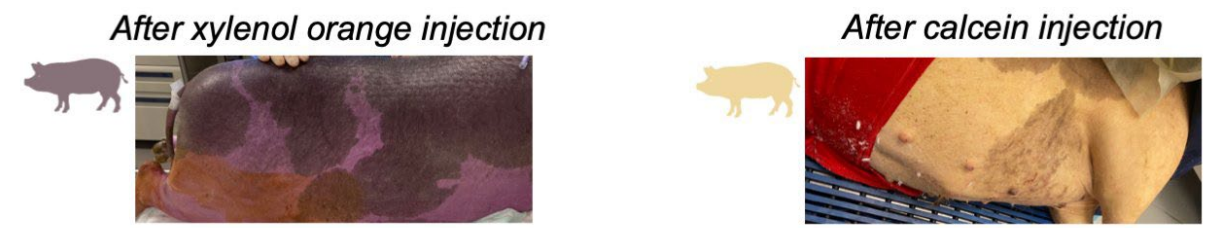

b

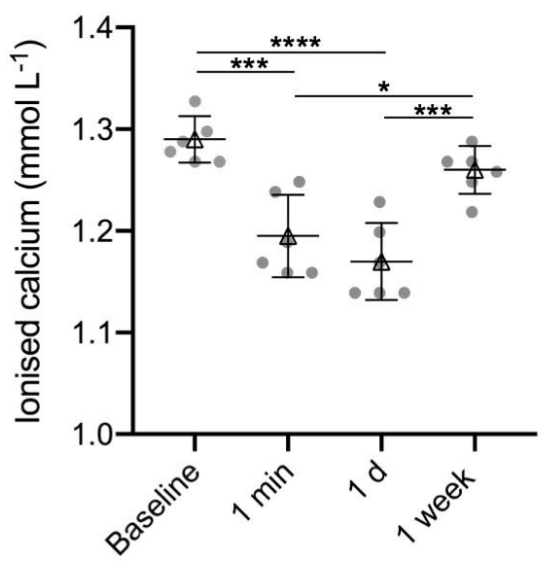

Time after xylenol orange injection

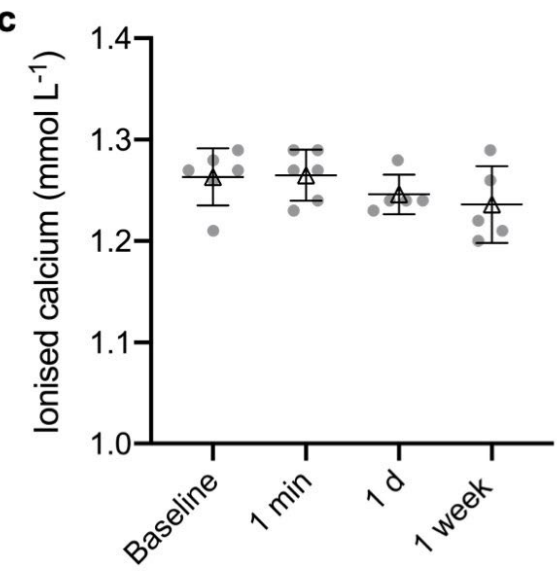

Time after calcein injection

Fig 2. Blood analyses before and after injecting fluorochrome labels. (a) Minipig skin colouring postfluorochrome injection. Immediately after xylenol orange injection, the animals turned purple. The animals turned slightly yellow after calcein injection. Animal colouring returned to normal within $3 \mathrm{~h}$ post-injection. $(\mathbf{b}, \mathbf{c})$ Ionised calcium levels in the blood after $(\mathbf{b})$ xylenol orange and (c) calcein injection through 2 weeks post-surgery. For both plots, individual animal data are represented by the circles. Triangles and error bars represent the mean \pm standard deviation. ${ }^{*} p<0.05$, ${ }^{* * *} p<0.001$, ${ }^{* * * *} p<0.0001 . N=5-6$ animals. 
the systemic distribution of the label. The xylenol orange and calcein injections caused the animals' skin to transiently turn purple and yellow, respectively. Animal skin coloration returned to normal within few hours post-injection. Blood samples were taken both pre- and post-injection ( $1 \mathrm{~min}, 1 \mathrm{~d}, 1$ week) and analysed for ionised calcium levels. There was a slight decrease in ionised calcium after the injection of xylenol orange label (Fig. 2b) but this returned to baseline within 1 week post-injection. After the calcein injection, there were no changes in ionised calcium (Fig. 2c).

\section{Mechanical testing and histology of regenerating cartilage}

Indentation testing was performed to assess the mechanical properties of the repair tissue (Fig. 3a). Native cartilage controls were taken from the distal portion of the operative femoral trochlea. As expected, all treatment groups had a significantly lower compressive modulus in comparison to the native cartilage controls (Fig. 3b). There were no differences observed between MST groups at this early time point (Fig. 3c). There were additionally no differences in the hydraulic permeability between treatment groups as well (data not shown). Similarly, histological assessment showed similar cartilage repair between treatment groups 4 weeks postsurgery. Faint outlines of the original MST holes were present in both SmartShot ${ }^{\circledR}$ conditions, whereas more prominent void spaces were visible in the K-wire group (Fig. 4a). Blinded ICRS II scoring revealed improved basal integration in the $0.9 \mathrm{~mm} \mathrm{SmartShot}{ }^{\circledR}$ group compared to the microfracture group (Fig. 4 b). There were no significant differences between treatment groups in the nine other parameters scored: defect fill, integration to surrounding cartilage, matrix staining, surface architecture, subchondral bone abnormality, vascularisation, surface/superficial assessment, mid/deep zone assessment and overall assessment. As evident from the representative SafO/ FG images, matrix staining was minimal across all groups.

\section{Fluorochrome imaging}

Fluorochrome label incorporation at the time of surgery (xylenol orange) and 2 weeks post-surgery (calcein) showed marked differences in the bony remodelling response for each MST technique (Fig. $5 a)$. For this analysis, a region of interest was set adjacent to the MST hole (Fig. 5b). The percent area of each mineral label was quantified within this region of interest. The defects treated with the SmartShot ${ }^{\circledR}$ devices had the highest xylenol orange incorporation, indicative of early bone formation, while a delayed response was seen in the K-wire group (Fig. 5c). At the later time point ( 2 weeks), the $\mathrm{K}$-wire group had significantly more calcein incorporation in comparison to all other groups (Fig. 5d). The defects treated with microfracture awl had relatively even percentages of each label, signifying a steady, but relatively quiet, bony response over the 4 weeks testing period.

\section{Global bony response}

$\mu \mathrm{CT}$ was used to assess bone resorption in the subchondral bone underlying the cartilage defects 4 weeks post-surgery (Fig. 6a). Similar to the histology findings, the MST holes were most apparent in the K-wire group. For each treatment group, the MST holes were excluded from the bone resorption measurements. 3D quantification of bone resorption indicated that the K-wire group had significantly more bone resorption compared to the $1.2 \mathrm{~mm}$
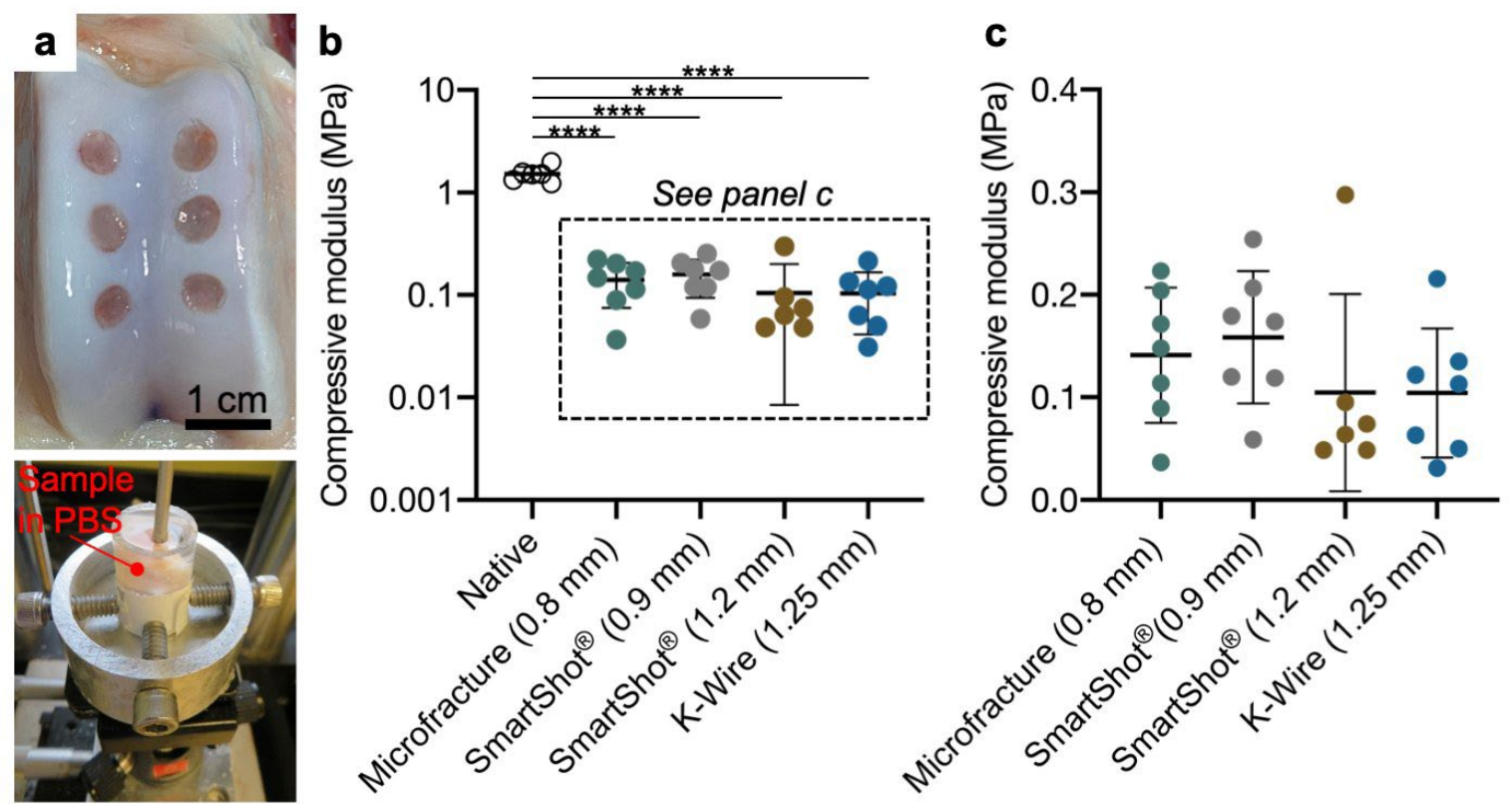

Fig 3. Macroscopic cartilage repair and cartilage mechanical properties. (a) Macroscopic image of joint 4 weeks post-surgery and indentation testing setup. (b) Compressive modulus (displayed on $\log _{10}$ scale) including the native cartilage controls. ${ }^{* * * *} p<0.0001$. (c) Compressive modulus (linear scale, from b). All plots show mean \pm standard deviation. $n=7$ defects/treatment. $N=6$ animals. 
SmartShot ${ }^{\circledR}$. Cryosections stained for TRAP, which is expressed by osteoclasts, revealed that there was more osteoclastic activity in the subchondral region of defects treated with the K-wire in comparison to both the $1.2 \mathrm{~mm} \mathrm{SmartShot}^{\circledR}$ and native bone (Fig. 6b).

\section{Bone compaction}

Immediately after euthanasia, the contralateral hind limbs were used to investigate the initial bone compaction caused by each MST device (Fig. 7a). For this time 0 assessment, chondral defects were created, treatment groups were randomised across defect locations and $\mu \mathrm{CT}$ imaging was performed on the osteochondral units after marrow access. Higher $\mathrm{BV} / \mathrm{TV}$ measurements represented increased bone compaction. The K-wire group showed a significantly higher BV/TV in the region immediately adjacent to the marrow access holes in comparison to both the
$0.9 \mathrm{~mm}$ SmartShot ${ }^{\circledR}$ device and native bone. The range of BV/TV values measured from each SmartShot ${ }^{\circledR}$ device overlapped with the native bone BV/TV range, unlike the microfracture and K-wire groups.

\section{Discussion}

The present study directly compared bony changes that occurred post-MST using three different methods of marrow access hole creation: awl-based microfracture, needle puncture $\left(\right.$ SmartShot $\left.^{\circledR}\right)$ and subchondral drilling (using a K-wire). Although not expected to show differences, the study also characterised the cartilage repair quality from each of these treatments at the 4 week terminal time point. The histological appearance of the repair tissue was similar between treatments, apart from the $0.9 \mathrm{~mm}$ a Microfracture $(0.8 \mathrm{~mm})$ SmartShot ${ }^{\circledR}(0.9 \mathrm{~mm})$

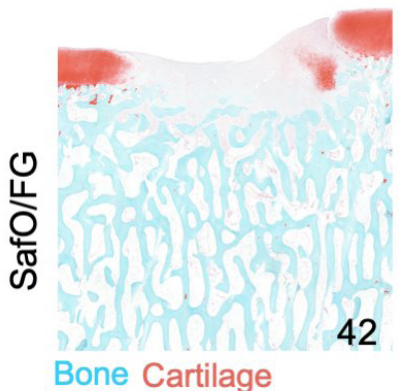

Bone Cartilage

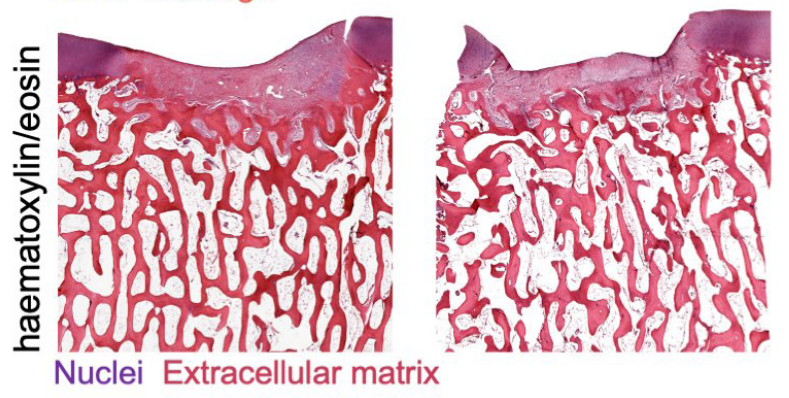

b

Basal integration

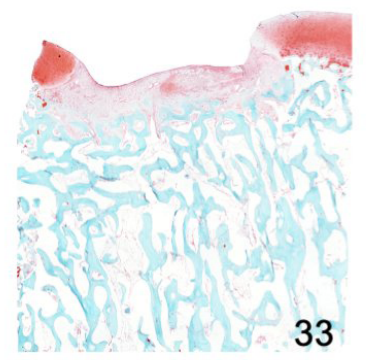

33
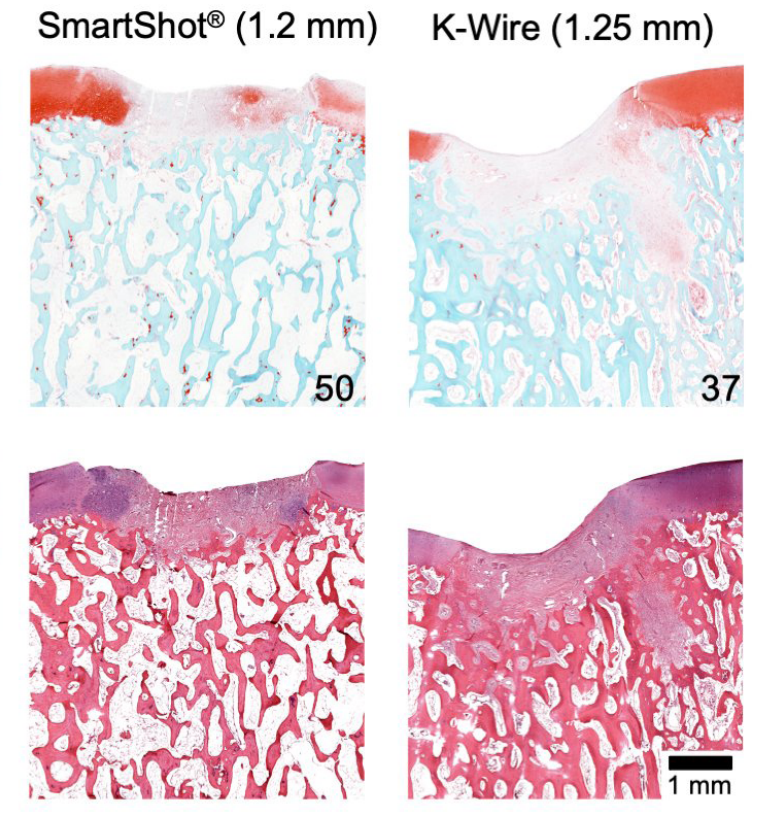

Defect fill

Overall assessment
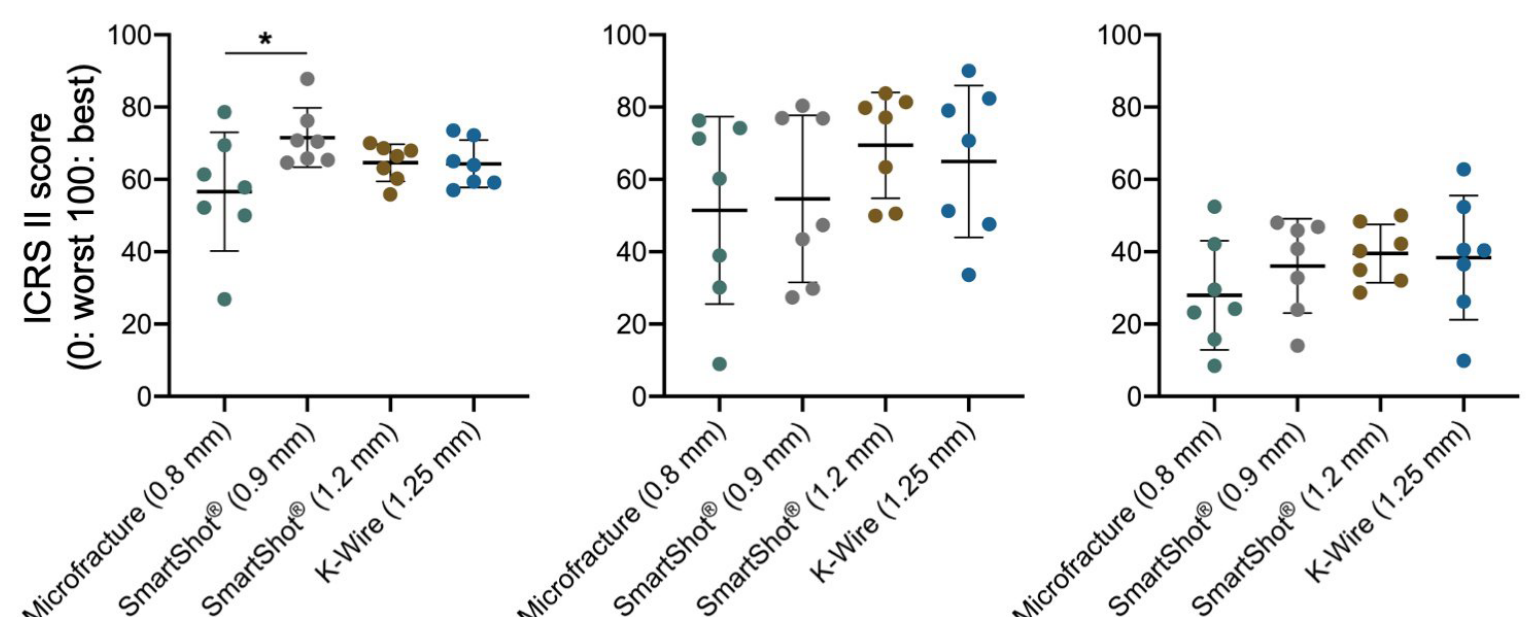

Fig 4. Cartilage repair quality 4 weeks after MST. (a) Histological staining (SafO/FG and haematoxylin/ eosin) of representative defects. Numbers represent the overall ICRS II histological score for that specimen. Limited proteoglycan deposition was observed in the defects at this early time point. (b) Blinded ICRS II histological scoring (0: worst, 100: best). ${ }^{*} p<0.05$. Bars indicate mean \pm standard deviation. $n=7$ defects/ treatment. $N=6$ animals. 
SmartShot ${ }^{\circledR}$ enhancing basal integration (integration between the underlying bone and cartilage repair tissue) in comparison to the microfracture group. Comprehensive analyses were performed to assess bone quality post-MST including, histology, $\mu \mathrm{CT}$ and fluorochrome labelling. Taken together, these results highlighted potential disadvantages in the use of K-wires for subchondral drilling. The $1.25 \mathrm{~mm}$ $\mathrm{K}$-wire caused the most bone resorption, delayed bony healing and compacted the bone around the perimeter of the MST holes in comparison to other marrow access methods. It may be that a fluted drill bit (Gao et al., 2018), designed to excise bone chips as the drill advances, would better preserve the subchondral bone in comparison to the smooth K-wire, however that was not assessed in the present study. A smaller diameter K-wire may have also caused less damage to the bone. Regardless, the $1.25 \mathrm{~mm} \mathrm{~K}$-wires used in this study are within the range commonly used in clinical practice. As an alternative approach, the SmartShot ${ }^{\circledR}$ device best preserved the subchondral bone. Interestingly, across all outcome measures, no statistical differences were found between the different sized SmartShot ${ }^{\circledR}$ devices, suggesting that the method of hole creation may play a larger role than hole size in terms of the post-MST bony response.

It is important to consider not only the hole diameter and puncture method but also the hole depth and number. All of these variables likely play a role in the subsequent bony healing and cartilage repair. In the present study, the microfracture awl had the shortest hole depth $(2.0 \mathrm{~mm})$ and the SmartShot ${ }^{\circledR}$ devices had the longest hole depth $(8.0 \mathrm{~mm})$, with the $\mathrm{K}$-wire in the middle (6.0 $\mathrm{mm}$ hole depth). Because the two SmartShot ${ }^{\circledR}$ devices had similar hole depths and identical methods of puncture, it was possible to more easily draw comparisons between these treatments. However, it should be noted that, given space constraints, 2 MST holes were created using the $1.2 \mathrm{~mm}$ diameter SmartShot ${ }^{\circledR}$ and $3 \mathrm{MST}$ were created using the $0.9 \mathrm{~mm}$ SmartShot ${ }^{\circledR}$. This difference in hole number may have led these conditions to be more similar across the tested outcomes.

Previous studies have highlighted the variability in MST, from the technique itself (Kroell et al., 2016; Theodoropoulos et al., 2012) to patient outcomes (Knutsen et al., 2007; Mithoefer et al., 2009). There is considerable intra- and inter-surgeon variability with MST (Kroell et al., 2016), which likely contributes to

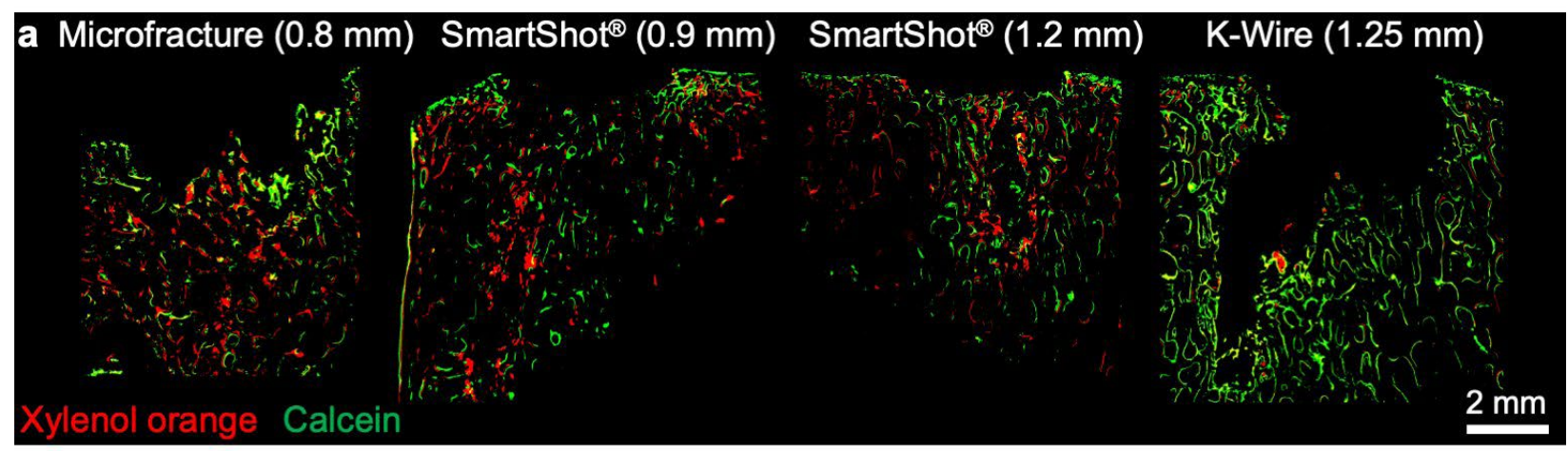

\section{b}

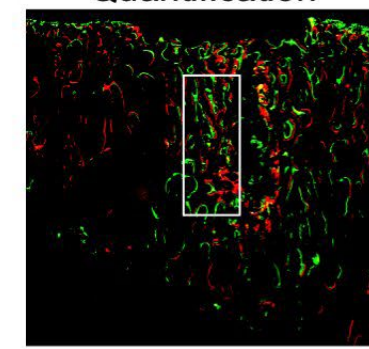

Xylenol

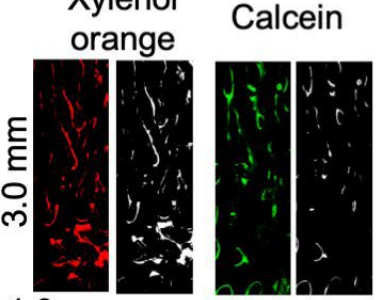

C

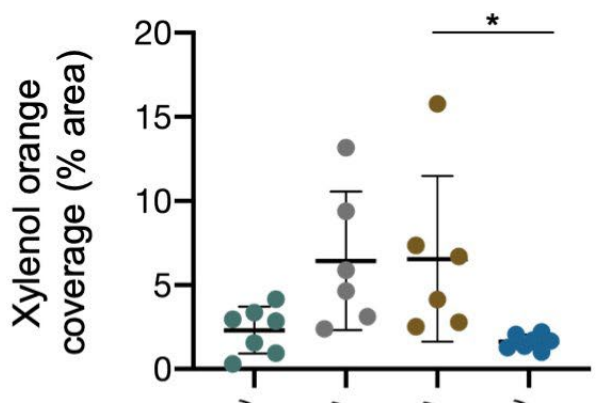

d

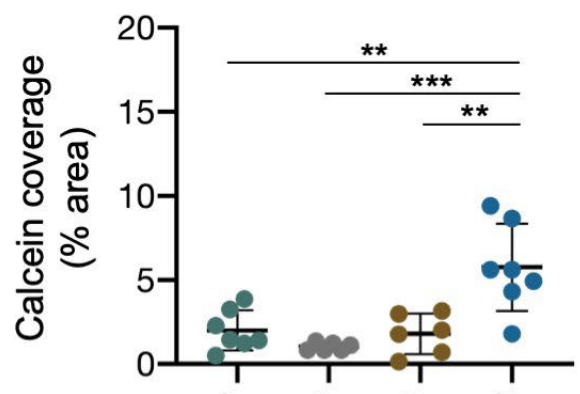

$1.0 \mathrm{~mm}$

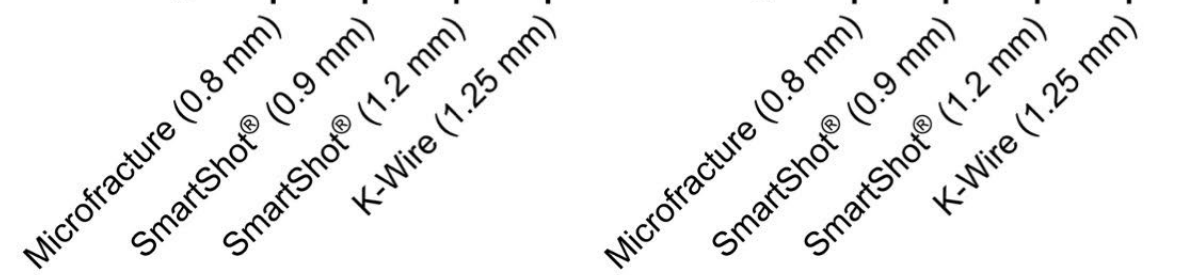

Fig 5. Bone remodelling over 4 weeks after MST. (a) Representative fluorochrome images at the midplane of the defects. Red: xylenol orange, injected at the time of surgery. Green: calcein, injected 2 weeks postsurgery. (b) Quantification of fluorochrome images. A $3.0 \mathrm{~mm}$ by $1.0 \mathrm{~mm}$ rectangular region nearest to the MST site was separated into the respective red and green channels and quantified. (c,d) Percentage area of xylenol orange and calcein, respectively. ${ }^{*} p<0.05,{ }^{* *} p<0.01,{ }^{* * *} p<0.001$. Bars indicate mean \pm standard deviation. $n=6-7$ holes/treatment. $N=6$ animals. 
the variable cartilage fill grade (18 to $95 \%$ ) (Mithoefer et al., 2009). While the focus of the present study was not on improving the repeatability of MST, results did support the use of the SmartShot ${ }^{\circledR}$ (or a similar device), which could offer greater control and uniformity of hole depth across surgeons. Further, the use of this device in both the preclinical and clinical procedures may enhance the repeatability of outcomes between research groups and surgical teams.

One of the primary advances of this study was the implementation of fluorochrome labelling in a large animal. The present is the first study that used fluorochrome imaging to monitor the dynamic bone remodelling that occurred after MST techniques. This longitudinal outcome measure reduces the number of animals required to visualise bony changes over time. While others have performed fluorochrome labelling in minipigs (Funk et al., 2009), this technique is relatively uncommon in large animals. The scarcity of large-animal studies utilising fluorochrome labelling may be due to the fact that while such mineral labels can be injected subcutaneously into small animals, large animals require intravenous administration (van Gaalen et al., 2010). Additionally, many of the fluorochrome labels used in rodents are not compatible with larger animals. Specifically, alizarine complexone can lead to sudden death in minipigs (Ruehe et al., 2008). To ensure animal safety over the duration of the study, periodic blood samples were taken from the intravenous catheter and the blood samples were analysed for ionised calcium. While there were statistically significant differences in ionised calcium levels after injecting xylenol orange, these transient changes were likely not physiologically significant a
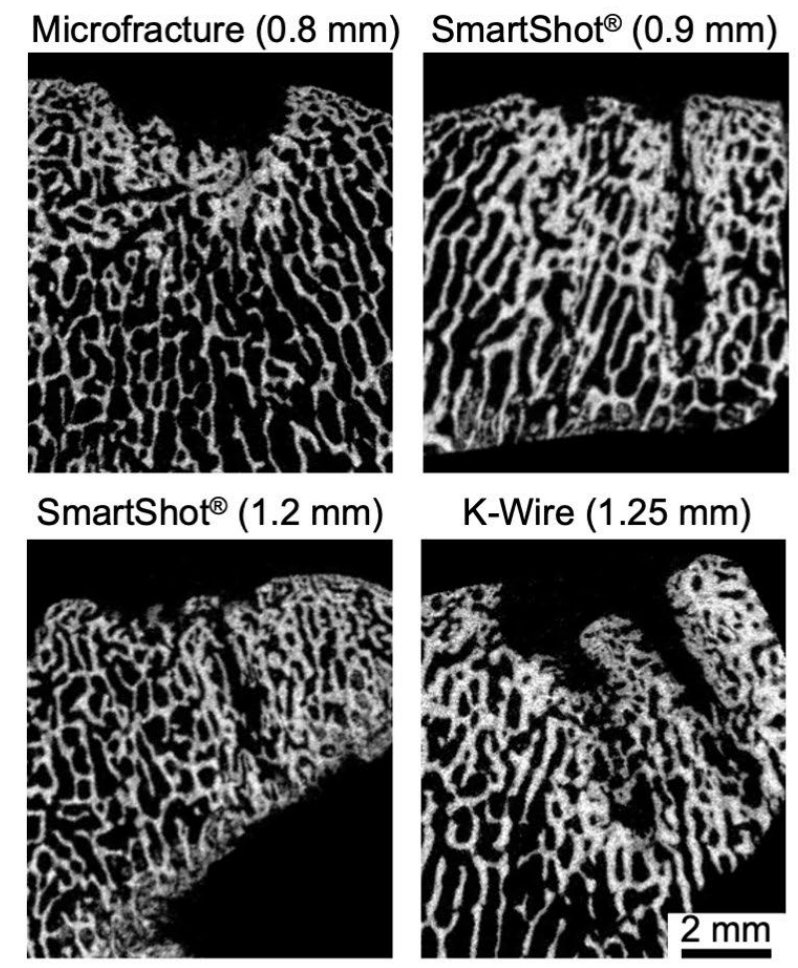

K-Wire $(1.25 \mathrm{~mm})$

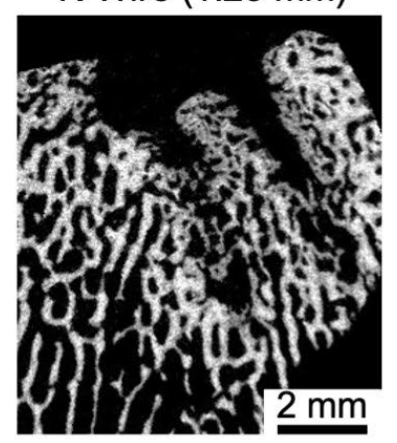

b
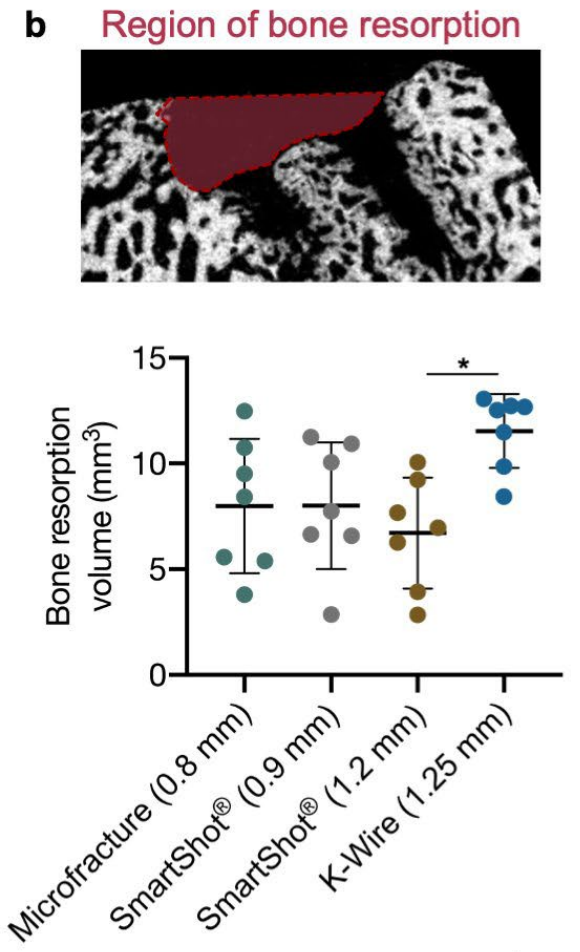
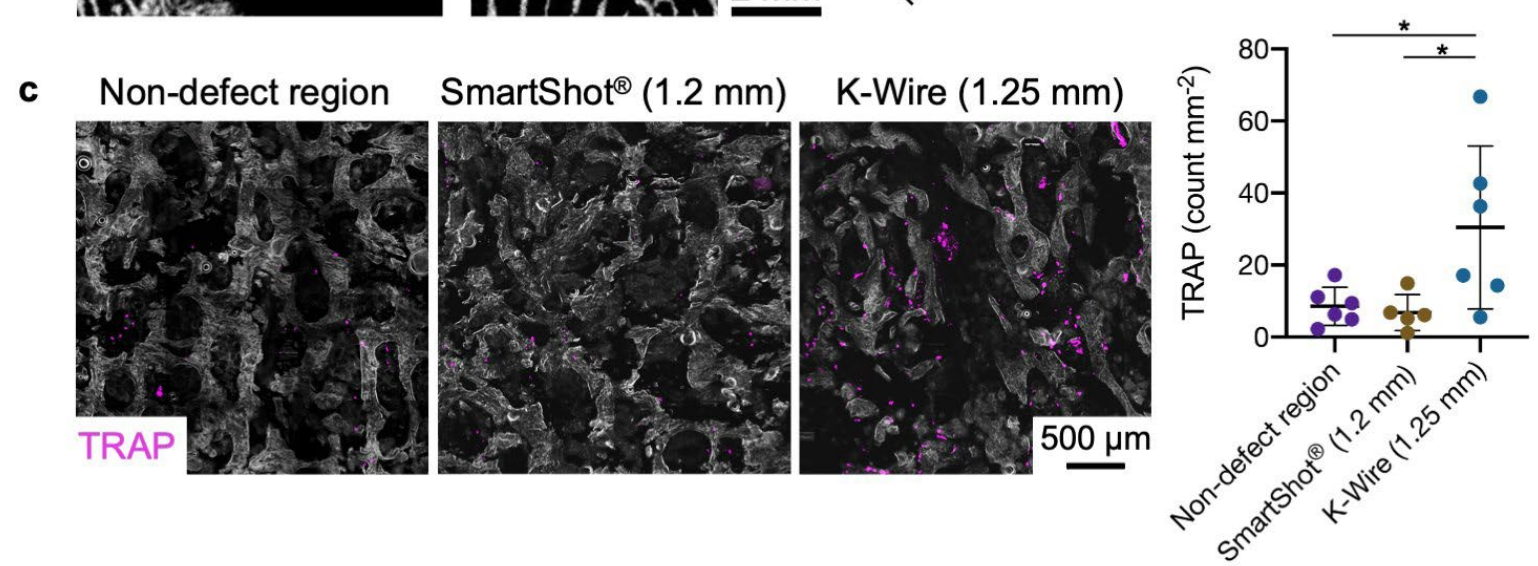

Fig 6. Global bony response underneath cartilage defects following MST. (a) Representative $\mu$ CT images at the midplane of the defects. (b) 3D quantification of bone resorption. ${ }^{*} p<0.05 . n=7$ defects/treatment. $N=6$ animals. (c) TRAP staining and quantification. TRAP positive pixels were normalised to the bone area in a $7 \mathrm{~mm}$ by $7 \mathrm{~mm}$ image. ${ }^{*} p<0.05$. Bars indicate mean \pm standard deviation. $n=5-6$ defects/treatment. $N=6$ animals. 
(that is, ionised calcium remained in the physiological range). Overall, the positive response of the animals to the described fluorochrome labelling protocol should encourage other groups to implement this potentially impactful outcome measure in studies of MST and osteochondral regeneration.

All large-animal studies are limited by sample size; nonetheless, an efficient utilisation of animals was demonstrated and significant differences were identified between treatment groups within the cohort, demonstrating the utility of study design to reduce animal numbers. Both the operative and non-operative hind limbs were used to establish two time points (time 0 and 4 weeks) for separate outcome measures. Additionally, both the cartilage repair quality and changes to the subchondral bone were assessed. While no differences in cartilage repair were expected at the 4 week time point (given how short the time period was), it was important to perform such assays to provide a baseline and motivation for future long-term studies evaluating next generation cell and material adjuvants to MST. Future work will also determine whether bone quality relates to repair tissue strength and durability.

\section{Conclusions}

This study was built on previous literature suggesting that small diameter and deeper MST holes may reduce subchondral bone abnormalities and ultimately

a

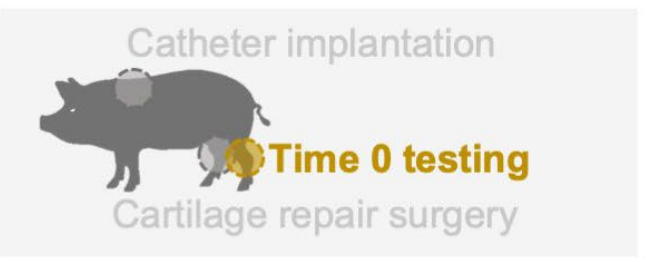

Time 0 testing

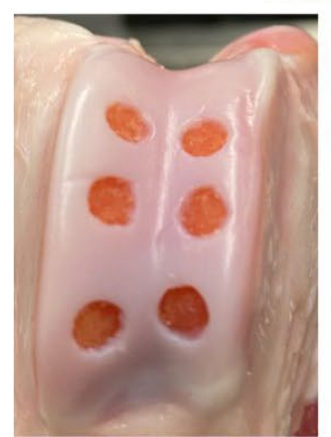

Defect creation

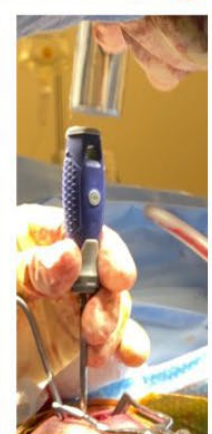

Treatment

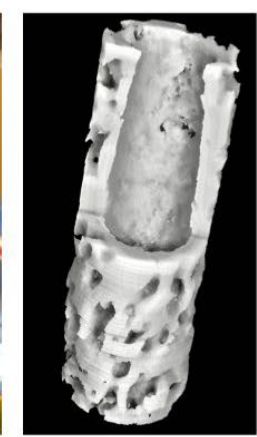

$\mu C T$

\section{Assess initial bone compaction} surrounding MST holes improve cartilage repair. While a few studies have compared tool sizing for MST, none have jointly investigated tool sizing and the method of hole creation. This study provided strong evidence in favour of a repeatable needle-puncture device (SmartShot ${ }^{\circledR}$ or a similar device) to best preserve the subchondral bone and minimise aberrant remodelling after MST. This work also provided a cautionary tale regarding the use of K-wires, as this treatment group consistently performed poorly with respect to subchondral bone remodelling and healing. Overall, the results of the study should inform the design of future preclinical studies and has the potential to change current surgical practice.

\section{Acknowledgements}

The study was supported by the NIH (R01 AR077362, T32 AR007132, P30 AR069619, F31 AR077395). Additional funding was provided by the Department of Veterans' Affairs and the NSF (CMMI: 1548571). The authors are grateful to the University of Pennsylvania's University Laboratory Animal Resources (ULAR) veterinary staff. Additionally, the authors would like to acknowledge Dr Sarah Gullbrand, Dr Sherry Liu, Dr Thomas Schaer, Dr Julia Funk and Dr Chelsea Wallace for their experimental guidance and advice. Lastly, the authors would like to thank Marrow Access Technologies for donating the SmartShot ${ }^{\circledR}$ devices and Dr Michael Hast for

b
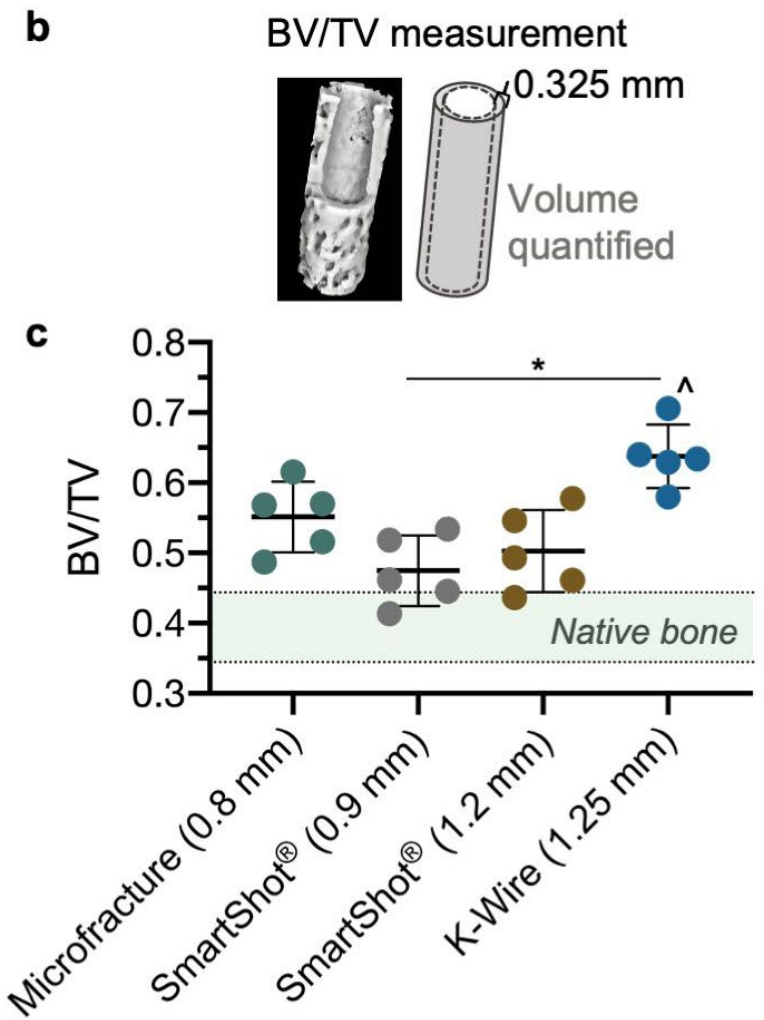

Fig 7. Bone compaction surrounding fresh (time 0) MST hole. (a) Schematic of time 0 testing. (b) BV/TV in region surrounding an MST hole. (c) Native bone was measured underneath a cartilage defect without any MST hole and the range of values are displayed by the shaded region. ${ }^{*} p<0.05 .{ }^{*} p<0.001$ in comparison to native bone. Bars indicate mean \pm standard deviation. $N=5$ holes/treatment. $N=4$ animals. 
donating the surgical drills used in this study. While no funding was accepted from Marrow Access Technologies for this study, Dr Jason Koh, a coauthor, serves as the Chief Medical Officer and owns stock in this company.

\section{References}

Bonadio MB, Friedman JM, Sennett ML, Mauck RL, Dodge GR, Madry H (2017) A retinaculumsparing surgical approach preserves porcine stifle joint cartilage in an experimental animal model of cartilage repair. J Exp Orthop 4: 11. DOI: 10.1186/ s40634-017-0083-7.

Chen H, Sun J, Hoemann CD, Lascau-Coman V, Ouyang W, McKee MD, Shive MS, Buschmann MD (2009) Drilling and microfracture lead to different bone structure and necrosis during bone-marrow stimulation for cartilage repair. J Orthop Res 27: 1432-1438.

Cole BJ, Farr J, Winalski CS, Hosea T, Richmond J, Mandelbaum B, De Deyne PG (2011) Outcomes after a single-stage procedure for cell-based cartilage repair: a prospective clinical safety trial with 2-year follow-up. Am J Sports Med 39: 1170-1179.

DiBartola AC, Everhart JS, Magnussen RA, Carey JL, Brophy RH, Schmitt LC, Flanigan DC (2016) Correlation between histological outcome and surgical cartilage repair technique in the knee: a meta-analysis. Knee 23: 344-349.

Dyment NA, Jiang X, Chen L, Hong SH, Adams DJ, Ackert-Bicknell C, Shin DG, Rowe DW (2016) High-throughput, multi-image cryohistology of mineralised tissues. J Vis Exp 2016: 1-11.

Eldracher M, Orth P, Cucchiarini M, Pape D, Madry H (2014) Small subchondral drill holes improve marrow stimulation of articular cartilage defects. Am J Sports Med 42: 2741-2750.

Funk JF, Krummrey G, Perka C, Raschke MJ, Bail HJ (2009) Distraction osteogenesis enhances remodeling of remote bones of the skeleton: a pilot study. Clin Orthop Relat Res 467: 3199-3205.

Gao L, Goebel LKH, Orth P, Cucchiarini M, Madry H (2018) Subchondral drilling for articular cartilage repair: a systematic review of translational research. Dis Model Mech 11: 1-12.

Gobbi A, Nunag P, Malinowski K (2005) Treatment of full thickness chondral lesions of the knee with microfracture in a group of athletes. Knee Surg Sports Traumatol Arthrosc 13: 213-221.

Heijink A, Gomoll AH, Madry H, Drobnič M, Filardo G, Espregueira-Mendes J, van Dijk CN (2012) Biomechanical considerations in the pathogenesis of osteoarthritis of the knee. Knee Surg Sports Traumatol Arthrosc 20: 423-435.

Jafarzadeh SR, Felson DT (2018) Updated estimates suggest a much higher prevalence of arthritis in united states adults than previous ones. Arthritis Rheumatol 70: 185-192.
Kim IL, Pfeifer CG, Fisher MB, Saxena V, Meloni GR, Kwon MY, Kim M, Steinberg DR, Mauck RL, Burdick JA (2015) Fibrous scaffolds with varied fiber chemistry and growth factor delivery promote repair in a porcine cartilage defect model. Tissue Eng Part A 21: 2680-2690.

Knutsen G, Drogset JO, Engebretsen L, Grøntvedt T, Isaksen V, Ludvigsen TC, Roberts S, Solheim E, Strand T, Johansen O (2007) A randomised trial comparing autologous chondrocyte implantation with microfracture: findings at five years. J Bone Joint Surg Am 89: 2105-2112.

Knutsen G, Isaksen V, Johansen O, Engebretsen L, Ludvigsen TC, Drogset JO, Grøntvedt T, Solheim E, Strand T, Roberts S (2004) Autologous chondrocyte implantation compared with microfracture in the knee: a randomised trial. J Bone Joint Surg Am 86: 455-464.

Kraeutler MJ, Aliberti GM, Scillia AJ, McCarty EC, Mulcahey MK (2020) Microfracture versus drilling of articular cartilage defects: a systematic review of the basic science evidence. Orthop J Sports Med 8: 1-7.

Kroell A, Marks P, Chahal J, Hurtig M, Dwyer T, Whelan D, Theodoropoulos J (2016) Microfracture for chondral defects: assessment of the variability of surgical technique in cadavers. Knee Surg Sports Traumatol Arthrosc 24: 2374-2379.

Madry H, Gao L, Rey-Rico A, Venkatesan JK, Müller-Brandt K, Cai X, Goebel L, Schmitt G, Speicher-Mentges S, Zurakowski D, Menger MD, Laschke MW, Cucchiarini M (2020) Thermosensitive hydrogel based on PEO-PPO-PEO poloxamers for a controlled in situ release of recombinant adenoassociated viral vectors for effective gene therapy of cartilage defects. Adv Mater 32: 1-8.

Mainil-Varlet P, Van Damme B, Nesic D, Knutsen G, Kandel R, Roberts S (2010) A new histology scoring system for the assessment of the quality of human cartilage repair: ICRS II. Am J Sports Med 38: 880-890.

Martín AR, Patel JM, Zlotnick HM, Carey JL, Mauck RL (2019) Emerging therapies for cartilage regeneration in currently excluded 'red knee' populations. NPJ Regen Med 4: 1-12.

Meloni GR, Fisher MB, Stoeckl BD, Dodge GR, Mauck RL (2017) Biphasic finite element modelling reconciles mechanical properties of tissue-engineered cartilage constructs across testing platforms. Tissue Eng Part A 23: 663-674.

Miller RE, Grodzinsky AJ, Barrett MF, Hung HH, Frank EH, Werpy NM, McIlwraith CW, Frisbie DD (2014) Effects of the combination of microfracture and self-assembling peptide filling on the repair of a clinically relevant trochlear defect in an equine model. J Bone Joint Surg Am 96: 1601-1609.

Mithoefer K, Mcadams T, Williams RJ, Kreuz PC, Mandelbaum BR (2009) Clinical efficacy of the microfracture technique for articular cartilage repair in the knee: an evidence-based systematic analysis. Am J Sports Med 37: 2053-2063.

Moore AC, DeLucca JF, Elliott DM, Burris DL (2016) Quantifying cartilage contact modulus, tension 
modulus, and permeability with hertzian biphasic creep. J Tribol 138: 1-7.

Morisset S, Frisbie DD, Robbins PD, Nixon AJ, Mcllwraith CW (2007) IL-1ra/IGF-1 gene therapy modulates repair of microfractured chondral defects. Clin Orthop Relat Res 462: 221-228.

Murphy L, Helmick CG (2012) The impact of osteoarthritis in the United States: a population health perspective: a population-based review of the fourth most common cause of hospitalisation in U.S. adults. Orthop Nurs 31: 85-91.

Orth P, Duffner J, Zurakowski D, Cucchiarini M, Madry H (2016) Small-diameter awls improve articular cartilage repair after microfracture treatment in a translational animal model. Am J Sports Med 44: 209-219.

Patel JM, Sennett ML, Martin AR, Saleh KS, Eby MR, Ashley BS, Miller LM, Dodge GR, Burdick JA, Carey JL, Mauck RL (2020) Resorbable pins to enhance scaffold retention in a porcine chondral defect model. Cartilage. DOI: 10.1177/1947603520962568.

Pfeifer CG, Fisher MB, Saxena V, Kim M, Henning EA, Steinberg DA, Dodge GR, Mauck RL (2017) Age-dependent subchondral bone remodelling and cartilage repair in a minipig defect model. Tissue Eng Part C Methods 23: 745-753.

Rahn BA, Perren SM (1971) Xylenol orange, a fluorochrome useful in polychrome sequential labeling of calcifying tissues. Stain Technol 46: 125129.

Riff AJ, Huddleston HP, Cole BJ, Yanke AB (2020) Autologous chondrocyte implantation and osteochondral allograft transplantation render comparable outcomes in the setting of failed marrow stimulation. Am J Sports Med 48: 861-870.

Ruehe B, Kershaw O, Niehues S, Nelson K (2008) Sudden death in miniature pigs. Lab Anim (NY) 37: 65-66.

Schindelin J, Arganda-Carreras I, Frise E, Kaynig V, Longair M, Pietzsch T, Preibisch S, Rueden C, Saalfeld S, Schmid B, Tinevez J-Y, White DJ, Hartenstein V, Eliceiri K, Tomancak P, Cardona A (2012) Fiji: an open-source platform for biologicalimage analysis. Nat Methods 9: 676-682.

Sennett M, Friedman J, Ashley B, Stoeckl B, Patel J, Alini M, Cucchiarini M, Eglin D, Madry H, Mata A, Semino C, Stoddart MJ, Moutos FT, Estes BT, Guilak F, Mauck R, Dodge G (2021) Long term outcomes of biomaterial-mediated repair of focal cartilage defects in a large animal model. Eur Cells Mater 41: 40-51.

Sharma B, Fermanian S, Gibson M, Unterman S, Herzka DA, Cascio B, Coburn J, Hui AY, Marcus N, Gold GE, Elisseeff JH (2013) Human cartilage repair with a photoreactive adhesive hydrogel composite. Sci Transl Med 5: 1-10.

Steadman JR, Briggs KK, Rodrigo JJ, Kocher MS, Gill TJ, Rodkey WG (2003) Outcomes of microfracture for traumatic chondral defects of the knee: average 11-year follow-up. Arthroscopy 19: 477-484.
Steadman JR, Rodkey WG, Briggs KK (2002) Microfracture to treat full-thickness chondral defects: surgical technique, rehabilitation, and outcomes. J Knee Surg 15: 170-176.

Theodoropoulos J, Dwyer T, Whelan D, Marks P, Hurtig M, Sharma P (2012) Microfracture for knee chondral defects: a survey of surgical practice among Canadian orthopedic surgeons. Knee Surg Sports Traumatol Arthrosc 20: 2430-2437. van Gaalen SM, Kruyt MC, Geuze RE, de Bruijn JD, Alblas J, Dhert WJA (2010) Use of fluorochrome labels in in vivo bone tissue engineering research. Tissue Eng Part B Rev 16: 209-217.

Volz M, Schaumburger J, Frick H, Grifka J, Anders $S$ (2017) A randomised controlled trial demonstrating sustained benefit of autologous matrix-induced chondrogenesis over microfracture at five years. Int Orthop 41: 797-804.

Zanotto G, Liebesny P, Barrett M, Zlotnick H, Grodzinsky A, Frisbie D (2019) Trypsin pre-treatment combined with growth factor functionalised selfassembling peptide hydrogel improves cartilage repair in rabbit model. J Orthop Res 37: 2307-2315.

Zedde P, Cudoni S, Giachetti G, Manunta ML, Masala G, Brunetti A, Manunta AF (2016) Subchondral bone remodelling: comparing nanofracture with microfracture. an ovine in vivo study. Joints 4: 87-93.

\section{Discussion with Reviewer}

Daniel Bisson: Between diameter, depth, reproducibility and puncture method parameters of MST, which do you think has the most influence on the outcome of the joint repair?

Authors: All of these factors - tool diameter, depth, reproducibility and puncture method - play a role in the outcome of joint repair. In the present study, surprising differences were found between puncture methods. For the most part, the $1.2 \mathrm{~mm}$ and $0.9 \mathrm{~mm} \mathrm{SmartShot}{ }^{\circledR}$ performed similarly, suggesting that hole diameter played less of a role than one might think. However, there are constraints on all these parameters. We would argue that within an acceptable range for hole diameter and hole depth (to prevent hole overlap), the puncture method has the most influence on the outcome of the joint repair. We did not directly focus on MST reproducibility, in part because a single surgeon performed all the surgeries. However, it would be interesting to study the inter- and intra-surgeon variability using different MST tools.

Editor's note: The Guest Editor responsible for this paper was Vaida Glatt. 